Bundesgesundheitsbl 2014 · 57:1002-1018 DOI 10.1007/s00103-014-2004-6

(c) Springer-Verlag Berlin Heidelberg 2014

Bekanntmachung des Umweltbundesamtes

\title{
Ermittlung und Beurteilung chemischer Verunreinigungen der Luft von Innenraumarbeits- plätzen (ohne Tätigkeit mit Gefahrstoffen)
}

\author{
Gemeinsame Mitteilung der \\ Arbeitsgruppe Luftanalysen der Ständigen \\ Senatskommission zur Prüfung gesundheits- \\ schädlicher Arbeitsstoffe der Deutschen \\ Forschungsgemeinschaft und der Ad-hoc- \\ Arbeitsgruppe Innenraumrichtwerte der \\ Kommission Innenraumlufthygiene und der \\ Obersten Landesgesundheitsbehörden
}

Die Bekanntmachung ist auch wie folgt veröffentlicht: Breuer D., Sagunski H. et al.: Ermittlung und Beurteilung chemischer Verunreinigungen der Luft von Innenraumarbeitsplätzen (ohne Tätigkeit mit Gefahrstoffen). In A. Hartwig (Hrsg.) Deutsche Forschungsgemeinschaft - Analytische Methoden zur Prüfung gesundheitsschädlicher Arbeitsstoffe, Luftanalysen, Bd. 1, Spezielle Vorbemerkungen, Wiley-VCH, Weinheim, 18. Lieferung, im Druck. http://onlinelibrary.wiley.com/ book/10.1002/3527600418/topics

\section{Einleitung}

Wohlbefinden, Gesundheit und Leistungsfähigkeit von Beschäftigten an Arbeitsplätzen in Gebäuden hängen von einer Reihe von Einflussfaktoren ab. Neben chemischen, physikalischen und biologischen spielen auch arbeitsphysiologische und psychologische Einflüsse eine Rolle. Chemische Faktoren umfassen
Gase, leicht- und schwerflüchtige organische Verbindungen, Partikel, Stäube und Staubinhaltsstoffe wie z. B. Metalle sowie Fasern. Gerüche werden den chemischen Einflüssen zugeordnet. $\mathrm{Zu}$ den physikalischen Faktoren zählen z. B. die Raumund Umgebungsflächentemperaturen, die Luftfeuchte, die Luftgeschwindigkeit, die Beleuchtung, eine blendfreie Umgebung, Geräusche (im Großraumbüro) bzw. Lärm (z. B. von Bürogeräten), elektromagnetische Felder, Radon und Ionen. Viren, Bakterien, Pilze, Haustierallergene und Pollen stellen die wesentlichen biologischen Faktoren dar. Außerdem sind die ergonomische Ausstattung, eine ungestörte Arbeitsumgebung und das soziale Arbeitsumfeld bei der Gesamtbetrachtung zu berücksichtigen. Dieser Beitrag bezieht sich ausschließlich auf die Bewertung möglicher chemischer Faktoren in Innenräumen.

Die im Folgenden beschriebenen Vorgehensweisen sollen Hinweise zur Be- wertung der Innenraumluft von solchen Arbeitsplätzen geben, an denen keine Tätigkeiten mit Gefahrstoffen durchgeführt werden. Diese Arbeitsplätze werden als Innenraumarbeitsplätze bezeichnet. Entsprechend gelten hier auch nicht die Arbeitsplatzgrenzwerte im Sinne der Gefahrstoffverordnung [1] wie z. B. AGW oder MAK-Werte [2, 3], sondern ein anderes Regelwerk an Beurteilungen (s. Abschn. 6 Beurteilungswerte).

Grundvoraussetzung für eine gute Innenraumluftqualität ist ein übliches $\mathrm{Ma} ß$ an Hygiene und Sauberkeit, wie z. B. die regelmäßige Reinigung der Arbeitsräume und der Oberflächen in Arbeitsräumen sowie eine Entleerung von Papierund Abfallkörben. Die Lüftung von Innenräumen gehört grundsätzlich zur üblichen Raumnutzung und ist den Raumnutzern zuzumuten. Eine nähere Bestimmung dieses zumutbaren Rahmens liefert seit Januar 2012 die Technische Regel ASR A3.6 „Lüftung“ zur Lüftung von 
Arbeitsstätten [4]. Danach muss in umschlossenen Arbeitsräumen gesundheitlich zuträgliche Atemluft vorhanden sein. Bei freier Lüftung soll in regelmäßigen Abständen nach Bedarf eine Stoßlüftung durchgeführt werden. Empfohlen wird eine drei- bis zehnminütige (Winter/ Sommer) Stoßlüftung alle $60 \mathrm{~min}$ (Büroraum) bzw. alle 20 min (Besprechungsraum). Beim Betrieb einer raumlufttechnischen Anlage wird eine regelmäßige Wartung und Pflege vorausgesetzt.

Hinsichtlich des Rauchens wird davon ausgegangen, dass das Rauchen an Arbeitsplätzen in Gebäuden in der Regel untersagt ist bzw. dass spezielle Raucherräume mit spezifischen Anforderungen eingerichtet sind.

\section{Definitionen}

\subsection{Innenräume}

Laut DIN EN ISO 16000-1 [5] gehören zu Innenräumen

- private Wohn- und Aufenthaltsräume,

- Räume in öffentlichen Gebäuden (z. B. Kindertagesstätten, Schulen, Jugendhäuser, Krankenhäuser, Sporthallen, Bibliotheken, Gaststätten und Veranstaltungsräume),

- Arbeitsräume und Arbeitsplätze in Gebäuden, die nicht gefahrstoffrechtlichen Regelungen unterliegen, sowie

- Innenräume von Kraftfahrzeugen und öffentlichen Verkehrsmitteln.

\subsection{Innenraumarbeitsplätze}

Entsprechend der Arbeitsstättenverordnung [6] sind Arbeitsräume solche Räume, in denen Arbeitsplätze innerhalb von Gebäuden dauerhaft eingerichtet sind. Solange dort keine Tätigkeiten mit Gefahrstoffen ausgeführt werden, werden diese als Innenraumarbeitsplatz betrachtet. $\mathrm{Zu}$ den Innenraumarbeitsplätzen zählen neben Büros auch Verkaufsräume sowie Arbeitsplätze in öffentlichen Gebäuden (z. B. Schulen, Kindergärten, Krankenhäuser, Sporthallen, Bibliotheken, Gaststätten, Theater und Kinos) [7]. Werden Tätigkeiten mit Gefahrstoffen ausgeführt, gilt die Gefahrstoffverordnung [1]. In diesem Fall greifen dann die in den Kapiteln der DFG-Methodensammlung Luftanalysen [8] beschriebenen Vorgehensweisen zur Ermittlung und Beurteilung der Belastungen gegenüber Gefahrstoffen.

Zwischen privaten Innenräumen und Innenraumarbeitsplätzen gibt es fließende Übergänge. So kann im Rahmen von Heim- und Telearbeit ein Raum im privaten Wohnbereich sehr wohl als Innenraumarbeitsplatz angesehen werden. Damit wird unter anderem die große Gruppe der Außendienstbeschäftigten erfasst, die im Regelfall über einen Arbeitsraum im eigenen Wohnbereich verfügen müssen.

$\mathrm{Zu}$ unterscheiden sind die Nutzungsphasen eines Innenraums (Raumnutzung ohne Tätigkeiten mit Gefahrstoffen) von gewerblichen Erstellungs-, Renovierungs- und Reinigungsphasen, in denen Tätigkeiten mit Gefahrstoffen durchgeführt werden. In solchen Fällen unterliegen diese Tätigkeiten der Gefahrstoffverordnung. Dazu gehören z. B. die Ausführung von Instandhaltungstätigkeiten wie Maler- und Fußbodenlegearbeiten oder Sanitärinstallationen.

\section{Stoffe in der Innenraumluft}

Luftverunreinigungen in Innenräumen können durch eine Vielzahl von Quellen hervorgerufen werden. $\bullet$ Tab. 1 gibt einen Überblick über mögliche Quellen und die wichtigsten von ihnen emittierten Stoffe.

\section{Messstrategie}

An Innenraumarbeitsplätzen kommt es immer wieder vor, dass Beschäftigte über Befindlichkeitsstörungen klagen, deren Ursache nicht klar ersichtlich ist. Angesichts der eingangs genannten Einflussfaktoren ist zunächst im Rahmen einer umfassenden Vorermittlung zu klären, ob chemische Einflüsse für die Beschwerden verantwortlich sein können. Neben chemischen und physikalischen Einflussfaktoren können auch psychische und andere Faktoren die Ursache sein. Diese sind nicht Gegenstand dieser Veröffentlichung. Umfangreiche Hilfestellungen für die Ermittlung und Beurteilung chemischer Verunreinigungen der Luft von Innenraumarbeitsplätzen gibt es $\mathrm{z}$. B. von den Unfallversicherungsträgern [7].
Damit im Rahmen von Innenraumluftmessungen repräsentative Messergebnisse erhalten werden, ist die Beschaffung umfassender Vorinformation erforderlich. Auf der Grundlage dieser Informationen wird in einem weiteren Schritt die Messstrategie abgeleitet. Hinweise zur Beschaffung der Vorinformation in Arbeitsbereichen liefert die TRGS 402 [9]. Bei Messungen in Innenräumen müssen zusätzlich die spezifischen Randbedingungen berücksichtigt werden, die für die Planung von Messungen in Innenräumen relevant sind. Diese werden in den Normenreihen DIN EN ISO 16000 [10] und VDI 4300 [11] beschrieben.

Bei Messungen in Innenräumen sind die klimatischen Bedingungen (Temperatur, Luftdruck und Luftfeuchte) zu erfassen und zu dokumentieren. Eine Korrektur der Ergebnisse auf Standardbedingungen $\left(20^{\circ} \mathrm{C}, 1013 \mathrm{hPa}\right)$ ist in Deutschland in der Regel nicht erforderlich, weil die in den Arbeitsräumen vorherrschenden Bedingungen nur unwesentlich von den Standardbedingungen abweichen.

\subsection{Vorermittlung}

Im Rahmen einer Vorermittlung ist die Besichtigung des Arbeitsplatzes z. B. durch eine Sicherheitsfachkraft, ein Messinstitut oder den betriebsärztlichen Dienst durchzuführen. Hilfestellung kann man auch bei den zuständigen Behörden und bei den Unfallversicherungsträgern erhalten. Lässt die Vorermittlung auf einen Einfluss chemischer Faktoren schließen, ist eine geeignete Messstrategie zu wählen. Aspekte für die Festlegung der Messstrategie an Innenraumarbeitsplätzen werden in den folgenden Abschnitten beschrieben.

Zur Informationsbeschaffung gehören neben einer Beschreibung des Raumes einschließlich der Baumaterialien und Einrichtungsgegenstände auch das Umfeld des zu überprüfenden Objektes sowie die Identifikation möglicher Quellen. Wichtig sind in diesem Zusammenhang auch Befragungen über Altlasten oder die Verwendung von Stoffen in benachbarten Bereichen. Auch die Raumtemperatur, Luftfeuchtigkeit und Luftbewegung sind für das Wohlbefinden von Bedeutung. Voruntersuchungen mit kontinu- 
Tab. 1 Quellen von Verunreinigungen der Luft an Innenraumarbeitsplätzen und die wichtigsten von ihnen emittierten Stoffe in Anlehnung an DIN EN ISO 16000 Blatt 1 [5]

\begin{tabular}{|c|c|c|c|}
\hline Quelle/Ursache & Vorgang/Aktivität & $\begin{array}{l}\text { Verwendete Produkte, Quellen } \\
\text { im engeren Sinne }\end{array}$ & Emittierte Stoffe \\
\hline \multicolumn{4}{|l|}{ Biologische Quellen } \\
\hline \multirow[t]{4}{*}{$\begin{array}{l}\text { Z. B. Menschen, Tiere, } \\
\text { Insekten, Milben }\end{array}$} & Atmung & & $\begin{array}{l}\text { Kohlendioxid, Wasserdampf, körpereigene Geruchsstoffe, } \\
\text { Geruchsstoffe aus Lebensmitteln }\end{array}$ \\
\hline & Transpiration & & Wasserdampf, Geruchsstoffe \\
\hline & $\begin{array}{l}\text { Verdauung, Ausscheidungs- } \\
\text { vorgang }\end{array}$ & & $\begin{array}{l}\text { Z. B. Geruchsstoffe, Ausscheidungen, Zersetzungspro- } \\
\text { dukte, Allergene, Endotoxine }\end{array}$ \\
\hline & Haarausfall, Hautabschilferung & & \\
\hline Zimmerpflanzen & $\begin{array}{l}\text { Ausdünstung, Schimmelpilz- } \\
\text { befall }\end{array}$ & & $\begin{array}{l}\text { Terpene und andere Geruchsstoffe, Wasserdampf, mikro- } \\
\text { bielle VOC }\end{array}$ \\
\hline \multicolumn{4}{|c|}{ Bausubstanz/Gebäudeausrüstung } \\
\hline $\begin{array}{l}\text { Baukörper und Bau- } \\
\text { materialien }\end{array}$ & $\begin{array}{l}\text { Produktverarbeitung, Ausga- } \\
\text { sung, Alterung, Abrieb, Zerset- } \\
\text { zung, Schimmelpilzbefall }\end{array}$ & $\begin{array}{l}\text { Baustoffe, Bauten- und Korro- } \\
\text { sionsschutzmittel, Isolierstoffe, } \\
\text { Dichtungsmaterialien, Farben, } \\
\text { Betonzusätze }\end{array}$ & $\begin{array}{l}\text { Verschiedenartige gas- und partikelförmige Stoffe, wie } \\
\text { z. B. Lösemittel, Weichmacher, Monomere, Oligomere, } \\
\text { Holz- und Flammschutzmittel, Fasern (Asbest, Mineral- } \\
\text { wolle), Radon (z. B. aus Granit), Amine und Ammoniak, } \\
\text { mikrobielle VOC }\end{array}$ \\
\hline $\begin{array}{l}\text { Raumlufttechnische } \\
\text { Anlagen }\end{array}$ & Betrieb und Wartung & $\begin{array}{l}\text { Wäscher, Filter, Isolier- und Dich- } \\
\text { tungsmaterialien, Ablagerungen, } \\
\text { Wärmetauscher }\end{array}$ & Biozide, Fasern, Geruchsstoffe \\
\hline $\begin{array}{l}\text { Raumausstattung, } \\
\text { Einrichtungsgegen- } \\
\text { stände }\end{array}$ & $\begin{array}{l}\text { Produktverarbeitung, Reno- } \\
\text { vierung, Ausgasung }\end{array}$ & $\begin{array}{l}\text { Möbel, Fußbodenbeläge, Heim- } \\
\text { textilien, Lacke und Anstrichmittel, } \\
\text { Tapeten }\end{array}$ & $\begin{array}{l}\text { Monomere und Oligomere aus Kunststoffen, Harzen, } \\
\text { Oberflächenbeschichtungen, Klebern (z. B. Formalde- } \\
\text { hyd), Fasern, Lösemittel, Weichmacher, Stabilisatoren, } \\
\text { Biozide (z. B. Pyrethroide) }\end{array}$ \\
\hline \multicolumn{4}{|c|}{ Aktivitäten im Innenraum } \\
\hline Nutzung als Büro & Bürobetrieb & Büroartikel, EDV-Geräte, Kopierer & $\begin{array}{l}\text { Organische Lösemittel, schwerflüchtige organische Stoffe } \\
\text { (Weichmacher, Flammschutzmittel), Druckeremissionen }\end{array}$ \\
\hline Raumpflege & $\begin{array}{l}\text { Reinigungs- und Pflegemaß- } \\
\text { nahmen, Schädlingsbekämp- } \\
\text { fung }\end{array}$ & $\begin{array}{l}\text { Wasch- und Putzmittel, Polituren, } \\
\text { Desinfektionsmittel, Schädlings- } \\
\text { bekämpfungsmittel }\end{array}$ & $\begin{array}{l}\text { Wasser, Ammoniak, Chlor, organische Lösemittel (z. B. } \\
\text { Ethanol), Bakterizide, Insektizide und Staub }\end{array}$ \\
\hline $\begin{array}{l}\text { Hygiene und Körper- } \\
\text { pflegemittel }\end{array}$ & $\begin{array}{l}\text { Körperpflege, kosmetische Be- } \\
\text { handlung }\end{array}$ & $\begin{array}{l}\text { Kosmetische Mittel und Bedarfs- } \\
\text { gegenstände }\end{array}$ & $\begin{array}{l}\text { Lösemittel, Treibgase, Duftstoffe, anorganische und orga- } \\
\text { nische Aerosole (Farbstoffe, Pigmente, Lacke, Harze) }\end{array}$ \\
\hline $\begin{array}{l}\text { Koch- und Heizvor- } \\
\text { gänge }\end{array}$ & $\begin{array}{l}\text { Verbrennungsprozesse (Hei- } \\
\text { zen, Kochen), Betrieb von of- } \\
\text { fenen Feuerstellen (z. B. auch } \\
\text { Kerzen) }\end{array}$ & $\begin{array}{l}\text { Kohle, Heizöl, Gas, Holz, Lebens- } \\
\text { mittel }\end{array}$ & $\begin{array}{l}\text { Gas (z. B. Stadtgas, Flaschengas, Erdgas), Heizöldampf, } \\
\text { Kohlendioxid, Kohlenmonoxid, Wasserdampf, Schweb- } \\
\text { staub, Kohlenwasserstoffe und viele andere organische } \\
\text { Stoffe (Verbrennungs-/Schwelprodukte) }\end{array}$ \\
\hline \multicolumn{4}{|l|}{ Außenluft } \\
\hline $\begin{array}{l}\text { Emissionen durch } \\
\text { menschliche Aktivi- } \\
\text { täten }\end{array}$ & $\begin{array}{l}\text { Lüftung, Infiltration und Diffu- } \\
\text { sion durch die Gebäudehülle } \\
\text { hindurch }\end{array}$ & $\begin{array}{l}\text { Gewerbe-/Industrie-Betriebe, Ver- } \\
\text { kehr, Hausbrand, Landwirtschaft, } \\
\text { Brände im Freien }\end{array}$ & $\begin{array}{l}\text { Anorganische und organische Gase und Aerosole, Löse- } \\
\text { mittel, Ammoniak, Geruchsstoffe, PAH }\end{array}$ \\
\hline $\begin{array}{l}\text { Biogene und geogene } \\
\text { Emissionen }\end{array}$ & $\begin{array}{l}\text { Lüftung, Eindringen von Bo- } \\
\text { denluft, Staubaufwirbelung }\end{array}$ & $\begin{array}{l}\text { Blühende Pflanzen, Boden, De- } \\
\text { ponien, Altlasten, Bodenresuspen- } \\
\text { sionen, natürliche Verrottung }\end{array}$ & $\begin{array}{l}\text { Pollen, Radon, Methan u. a. flüchtige organische Verbin- } \\
\text { dungen (Kohlenwasserstoffe, Organohalogenverbindun- } \\
\text { gen), Geruchsstoffe, Stäube }\end{array}$ \\
\hline Lebewesen & Ausscheidungen & $\begin{array}{l}\text { Darmgase, Geruchsstoffe und Aus- } \\
\text { scheidungs-/Zersetzungsprodukte }\end{array}$ & $\begin{array}{l}\text { Methan, Ammoniak und Schwefelverbindungen und bio- } \\
\text { logische Agenzien }\end{array}$ \\
\hline
\end{tabular}

ierlich registrierenden Messgeräten wie Flammenionisationsdetektoren (FID) oder Photoionisationsdetektoren (PID) können wertvolle Hinweise über das Vorhandensein einer Quelle geben. Im Einzelnen sind dabei Antworten auf folgende Fragen zu finden:

- Sind Verdachtsmomente für luftgetragene Verunreinigungen in dem Raum oder in benachbarten Bereichen vorhanden?
Hierzu zählen Einzelstoffe, Stoffgemische und Zubereitungen.

- Gibt es im Gebäude Räume und Bereiche, in denen nicht über Beschwerden berichtet wird? Sind diese Räume/Bereiche vergleichbar ausgestattet?

- Befinden sich Emissionsquellen in dem Raum?

Dazu gehören z. B. Einrichtungsgegenstände, Bürogeräte/-materialien.
Bauseitige Quellen: Emissionen durch Bauprodukte (für Hinweise, welche Stoffe aus Baumaterialien freigesetzt werden können, s. [12])

Bauseitig sind sowohl offene als auch verdeckte Emissionsquellen zu berücksichtigen.

- Welche Emissionscharakteristik weist die Quelle auf? (Beispiele siehe - Abb. 1) 

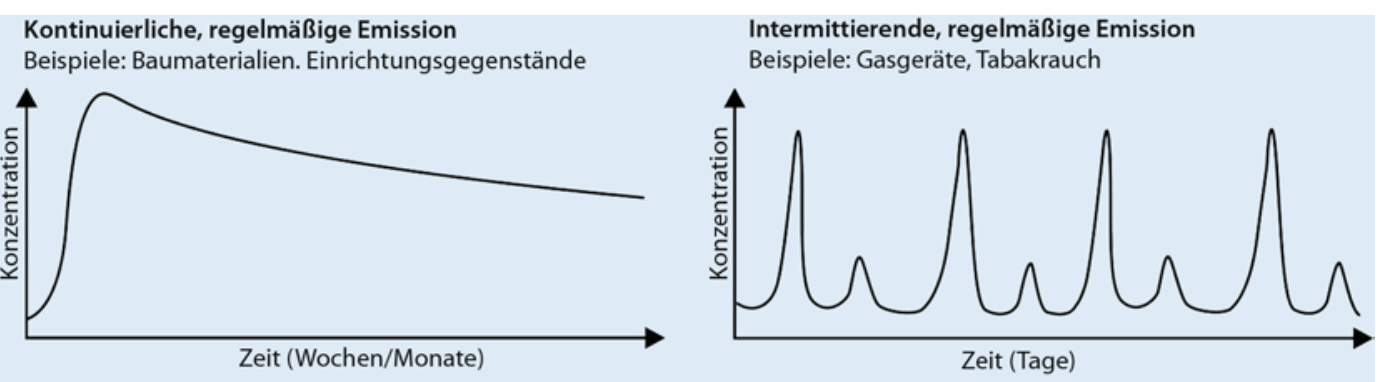

Kontinuierliche, unregelmäßige Emission

Beispiele: Farben, Lacke, Kleber bei Renovierungsarbeiten



Intermittierende, unregelmäßige Emission Beispiele: Schädlingsbekämpfungsmittel, Hobbyprodukte

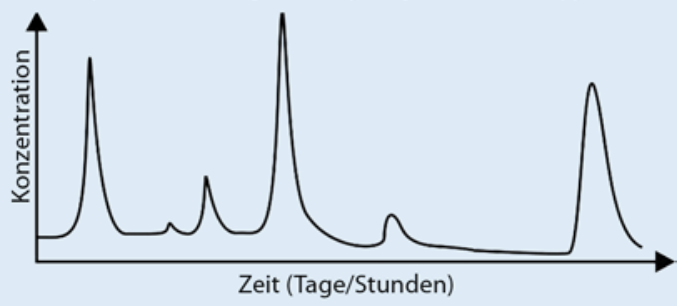

Abb. $1<$ Emissionscharakteristika einiger Quellen für Luftverunreinigungen in Innenräumen [13]
Kontinuierliche Quellen: z. B. Baumaterialien oder Einrichtungsgegenstände, die Stoffe über einen längeren Zeitraum freisetzen

Diskontinuierliche Quellen: z. B. der Gebrauch von Reinigungs- und Pflegemitteln oder auch von Schädlingsbekämpfungsmitteln

- Sind in jüngster Vergangenheit Renovierungs- und/oder Instandsetzungsarbeiten vorgenommen worden?

Sowohl Außenarbeiten, wie z. B. Hydrophobierung des Gebäudes, als auch Innenarbeiten, wie z. B. in den Raum eingebrachte Lacke, Farben, Klebstoffe usw., können typische kontinuierliche Quellen darstellen.

- Sind Maßnahmen zur energetischen Sanierung durchgeführt worden?

Dazu gehören insbesondere die Wärmedämmung oder der Austausch von Fenstern. Diese Maßnahmen beeinflussen den Luftwechsel und damit das Raumklima.

- Welche Historie haben das Gebäude und der Raum?

Dazu sind Informationen über das Alter und die Nutzung des Gebäudes sowie mögliche Havarien (z. B. Brandschäden) einzuholen. Auch Kenntnisse über das Grundstück (wenn der Untergrund möglicherweise eine Altlastenverdachtsfläche darstellt) sollten dabei berücksichtigt werden.

- Sind Quellen in der unmittelbaren Nachbarschaft, insbesondere durch gewerbliche Nutzung, im gleichen Gebäude vorhanden?

Dazu gehören z. B. Produktionsanlagen, Lagerbereiche oder Dienstleister (z. B. chemische Reinigung, Restaurant) im gleichen Gebäude.

- Spielen Emissionen in der Außenluft möglicherweise eine Rolle für die Innenraumluftqualität?

Dazu zählen Emissionen des Straßenverkehrs wie $\mathrm{CO}, \mathrm{NO}_{\mathrm{x}}, \mathrm{CO}_{2}$ und Partikel (Ruß) sowie andere Emittenten, wie z. B. Industrieanlagen, Abfallbeseitigungsanlagen oder landwirtschaftliche Betriebe.

- Wie erfolgt die Raumlüftung?

Dabei ist zwischen natürlicher und technischer Lüftung zu unterscheiden. Bei RLT-Anlagen sind z. B. die Luftwechselrate oder andere eingestellte Parameter wie die Luftfeuchte $\mathrm{zu}$ berücksichtigen.

- Wie ist der zeitliche Verlauf der Beschwerden bei den Beschäftigten?

Treten die Symptome z. B. täglich, nach Arbeitsende, nur an speziellen Arbeitstagen, morgens oder beim Betreten des Raumes auf? Hier kann als Schwierigkeit für die Messplanung der Umstand auftreten, dass Exposition und Wirkungseintritt zeitlich weit auseinanderliegen.

Die im Anhang dargestellte Checkliste erleichtert die Dokumentation der erhaltenen Vorinformation.

\subsection{Planung der Messung}

Auf Grundlage der Ergebnisse der Vorermittlung wird ein Messplan erstellt und mit dem Auftraggeber abgestimmt. Der Messplan umfasst folgende Fragestellungen:

- Warum soll gemessen werden (Grund)?

- Was soll gemessen werden (Stoff/ Stoffgruppe)?

- Wann soll gemessen werden (Zeitpunkt)?

- Wo soll gemessen werden (Messorte)?

- Wie soll gemessen werden (Messverfahren)?

\subsubsection{Warum soll gemessen werden?}

Haben die vorab gestellten Fragen Verdachtsmomente auf luftgetragene Verunreinigungen ergeben, so wird die Durchführung von Messungen empfohlen. Die Messungen dienen dem Ziel, Art, Höhe und Umfang der Exposition festzustellen, um auf dieser Grundlage über die Notwendigkeit von Maßnahmen zu entscheiden. Werden auf Basis der Messungen Maßnahmen (z. B. Sanierungen) durchgeführt, wird empfohlen, deren Erfolg durch eine Wirksamkeitskontrolle zu überprüfen.

\subsubsection{Was soll gemessen werden?}

Die Antwort auf die Frage nach den Stoffen bzw. den Stoffgruppen, die analysiert 


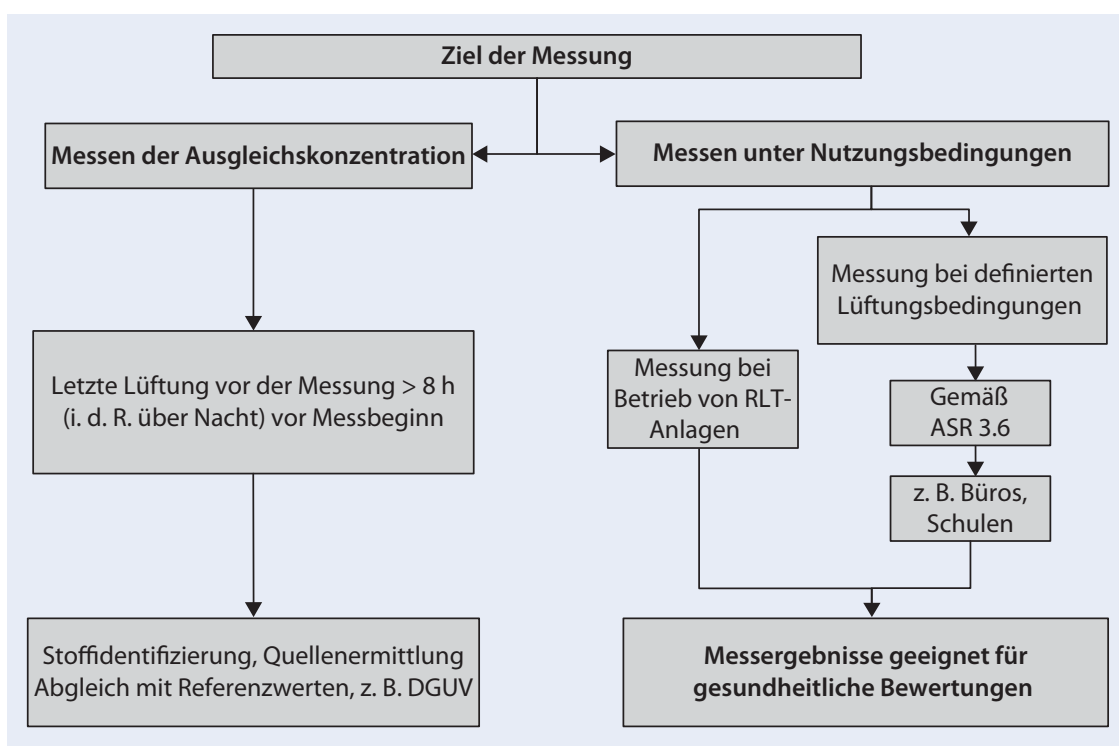

Abb. $2 \Delta$ Übersicht über die unterschiedlichen Messbedingungen

werden sollen, ergibt sich aus der Recherche, die im Vorfeld durchgeführt worden ist. Liegen keine oder nur unzureichende Informationen über die zu analysierenden Stoffe vor, ist es sinnvoll, eine Übersichtsanalyse über ein eingegrenztes Stoffspektrum, wie z. B. flüchtige organische Verbindungen (VOC) und Aldehyde, durchzuführen. In Räumen mit höherer Belegungszahl sollte darüber hinaus zur Beurteilung der Raumluftqualität auch Kohlendioxid $\left(\mathrm{CO}_{2}\right)$ bestimmt werden.

\subsubsection{Wann soll gemessen werden?}

Der Zeitpunkt der Messung ist abhängig von der Charakteristik der Quelle, der Raumlüftung, den Aktivitäten der Beschäftigten sowie der Raumtemperatur und der relativen Luftfeuchte. Bei diskontinuierlich kurzzeitig emittierenden Quellen ist eine Messung zur Ermittlung von Konzentrationsspitzen nur dann sinnvoll, wenn die Quelle aktiv ist. Auch die Lüftungsvorgaben und das Lüftungsverhalten der Beschäftigten müssen bei der Auswahl des Zeitpunktes berücksichtigt werden.

\subsubsection{Wo soll gemessen werden?}

Es wird empfohlen, je $50 \mathrm{~m}^{2}$ Grundfläche einen Messpunkt vorzusehen, dabei sind die räumlichen Gegebenheiten (z. B. Fensterfront, Türen, Verwinkelungen im Raum) zu berücksichtigen. Es sollte ortsfest gemessen werden. Die Messpunkte sind jedoch so zu wählen, dass die personenbezogene Exposition ermittelt wird.
Die Probenahmeeinrichtung sollte dabei in mindestens $1 \mathrm{~m}$ Entfernung von der Wand und in einer Höhe von mindestens $1 \mathrm{~m}$ aufgestellt werden. Vergleichsmessungen in einem nicht auffälligen Raum sind zu empfehlen. Kann ein Einfluss der Außenluft nicht ausgeschlossen werden, sollte auch eine Außenluftmessung erfolgen.

\subsubsection{Wie soll gemessen werden?}

Das auszuwählende Analysenverfahren muss dem zu analysierenden Stoff und der zu erwartenden Konzentration angepasst sein. Für Messungen über Zeiträume bis zu einigen Stunden kommen vorwiegend aktive Probenahmeverfahren, die aus einer Probenahmepumpe und einem anreichernden Probenträger bestehen, zum Einsatz. Bei längerer Probenahmedauer können ggf. auch andere Probenahmesysteme, wie z. B. Diffusionssammler, geeignet sein.

\subsection{Messung unter Nutzungsbedingungen}

Die seit Januar 2012 geltende ASR A3.6 [4] gibt Empfehlungen zur Lüftung von Innenraumarbeitsplätzen und enthält indirekt Vorgaben zur Messstrategie. Bei Messungen unter Berücksichtigung der Lüftungsempfehlungen nach ASR A3.6 werden nach vorangegangener intensiver Lüftung [ 3 min (Winter) bis $10 \mathrm{~min}$ (Sommer)] die Fenster und Türen ge- schlossen. Die Messung erfolgt nach $1 \mathrm{~h}$. Bei einer Messdauer von bis zu $1 \mathrm{~h}$ soll der Raum weiterhin geschlossen bleiben. Bei Langzeitmessungen $(t>1 \mathrm{~h})$ wird die Messung unter Einhaltung der empfohlenen Raumlüftung gemäß ASR A3.6 bei üblicher Nutzung durchgeführt. Die auf diese Weise erhaltenen Messergebnisse sind zum Vergleich mit Richtwerten [14] geeignet.

Bemerkung: Sind ablaufbedingt, wie z. B. in Schulen, kürzere Nutzungszyklen die Regel, so sollte die Messstrategie entsprechend angepasst werden.

Bei der Untersuchung von Räumen, die über eine raumlufttechnische Anlage (RLT-Anlage) belüftet werden, ist die Anlage vor der Probenahme mindestens $3 \mathrm{~h}$ lang bei den für den Raum üblichen Betriebsbedingungen zu betreiben.

\subsection{Messung unter Ausgleichsbedingungen}

Ist es notwendig, zusätzliche Informationen zu Quellen im Raum zu erhalten, sollte die Messung unter Ausgleichsbedingungen gemäß Messstrategie $[10,11]$ erfolgen. Dazu ist der Raum zunächst gründlich zu lüften und anschließend mindestens $8 \mathrm{~h}$ lang (in der Regel über Nacht) verschlossen zu halten. Die Probenahme erfolgt im Anschluss im ungelüfteten Raum. Mit dieser Messstrategie wurden unter anderem die Innenraumarbeitsplatz-Referenzwerte der DGUV [15] abgeleitet.

\subsection{Messstrategie für Arbeitsplätze}

Für Arbeitsplätze wird empfohlen, beide Messungen in direkter Abfolge durchzuführen (•Abb. 2). Zunächst erfolgt dabei die Messung unter Ausgleichsbedingungen und im Anschluss daran erfolgt die Messung unter Nutzungsbedingungen. Langzeitmessungen dürfen die für die zu beurteilenden Arbeitsplätze relevante Schichtlänge nicht überschreiten.

\section{Beispiele \\ Beseitigung einer bauseitigen Quelle}

Ein Gebäude in konventioneller Bauweise ohne Kellergeschoss, das in den 50er-Jahren errichtet wurde, ist in den 90er-Jahren umfassend gedämmt wor- 


\begin{tabular}{ll}
$\begin{array}{l}\text { Tab. } 2 \text { Beispiele für die Angabe von Mess- } \\
\text { ergebnissen }\end{array}$ \\
\begin{tabular}{ll} 
Messwert & Ergebnisangabe \\
\hline $1,22 \mathrm{mg} / \mathrm{m}^{3}$ & $1,2 \mathrm{mg} / \mathrm{m}^{3}$ \\
\hline $0,116 \mathrm{mg} / \mathrm{m}^{3}$ & $\begin{array}{l}0,12 \mathrm{mg} / \mathrm{m}^{3} \text { oder } \\
120 \mu \mathrm{g} / \mathrm{m}^{3}\end{array}$ \\
\hline $0,0122 \mathrm{mg} / \mathrm{m}^{3}$ & $\begin{array}{l}0,012 \mathrm{mg} / \mathrm{m}^{3} \text { oder } \\
12 \mu \mathrm{g} / \mathrm{m}^{3}\end{array}$ \\
& \\
\hline
\end{tabular} \\
\hline
\end{tabular}

den. Daraufhin wurden im Erdgeschoss Feuchtigkeitsschäden an den Wänden beobachtet. Zur Behebung des Schadens wurde der Baukörper im Fundamentbereich mit einer Wassersperrschicht versehen. Dazu wurde ein in Kohlenwasserstoffen gelöster Kunststoff ins Mauerwerk eingebracht. Nach Angaben des Produktdatenblattes sollte das Lösemittel innerhalb weniger Wochen verdunsten. Dennoch klagten einige der Beschäftigten auch 10 Wochen nach Einbringung der Wassersperrschicht über Reizungen der Atemwege. Eine in einem Raum durchgeführte Übersichtsmessung mit einem Diffusionssammler ergab eine TVOC-Konzentration von $55.000 \mu \mathrm{g} / \mathrm{m}^{3}$, die im Wesentlichen aus aliphatischen Kohlenwasserstoffen bestand. Dieses Ergebnis ist bei der Messung einer unabhängigen Messstelle bestätigt worden. Um die Absenkung der Schadstoffbelastung zu beschleunigen, wurden eine Erhöhung der Raumtemperatur und regelmäßiges Stoßlüften empfohlen. Eine nach 12 Wochen unter vergleichbaren Bedingungen durchgeführte Kontrollmessung bestätigte den Erfolg der Maßnahme; es wurde eine TVOC-Konzentration unter $1000 \mu \mathrm{g} / \mathrm{m}^{3}$ ermittelt.

Sanierung eines Bürokomplexes nach unsachgemäßem Bodenbelagseinbau

Im ersten Obergeschoss eines Verwaltungsneubaus klagten nahezu alle Beschäftigen bereits beim Betreten der Räume über Kopfschmerzen, Augen-, Schleimhaut- und Atemwegsreizungen. Im Erdgeschoss hingegen traten nur vereinzelt Beschwerden auf. Nach intensiver Recherche kristallisierten sich 2 Faktoren für das geplante Messprogramm als wesentliche Aspekte heraus. Einerseits musste der Bodenbelag in den Räumen des ersten Obergeschosses im Gegensatz zum Erdgeschoss aus zeitlichen Gründen ohne Protokollierung der Estrichrestfeuchte eingebaut werden. Andererseits wurde ein Kleber verwendet, der im alkalischen Mi- lieu zur Abspaltung von flüchtigen Komponenten neigt. Die Untersuchung der Raumluft von 6 Räumen, die hinsichtlich ihrer Ausstattung und Lage repräsentativ für den Gebäudekomplex waren, sowie von 2 identisch ausgestatteten Referenzräumen im Erdgeschoss bestätigte diesen Anfangsverdacht. Unter Nutzungsbedingungen wurden in den „Verdachtsräumen“ im Mittel 15.000 $\mu$ g 2-Ethyl-1-hexa$\mathrm{nol} / \mathrm{m}^{3}$ sowie weitere VOC gemessen. In den unbelasteten Vergleichsräumen lag die Konzentration an VOC bei $350 \mu \mathrm{g} / \mathrm{m}^{3}$. Anschließende Materialuntersuchungen des Fußbodenaufbaus (Teppich-KleberEstrich-Dämmung) identifizierten diesen als Quelle der Raumluftbelastung. Auf der Grundlage der Untersuchungsergebnisse wurde der Bodenbelag nebst Kleber und Estrich entfernt und erneuert. Kontrollmessungen nach Ende der Sanierung bestätigten den Erfolg der Maßnahme.

\section{Messverfahren}

\subsection{Grundlegende Feststellungen}

Bei der Messung von Innenraumluftverunreinigungen sind zahlreiche, zum Teil höchst unterschiedliche Stoffe und Stoffgruppen zu berücksichtigen. Diese können sowohl gas-/dampfförmig als auch partikulär auftreten. Bei der Wahl der Messverfahren ist darauf zu achten, dass sie für die Messung in Innenräumen geeignet sind.

Bei Messverfahren in Innenräumen, die als Arbeitsplätze genutzt werden, sind die Anforderungen in Anlehnung an TRGS 402 [9], DIN EN 482 [16] und die weiterführenden Normen DIN EN 838 [17], DIN EN 1076 [18], DIN EN 13890 [19] und DIN EN 13936 [20] zu berücksichtigen.

Für Arbeitsplätze, an denen Tätigkeiten mit Gefahrstoffen durchgeführt werden, hat ein Messverfahren gemäß DIN EN 482 zumindest einen Mindestmessbereich von einem Zehntel bis zum Zweifachen des Grenzwertes abzudecken. Diese Anforderung sollten auch Messverfahren erfüllen, die an Innenraumarbeitsplätzen eingesetzt werden. Zur Bewertung von Einzelstoffen in Innenräumen gibt es einen Vorsorgewert (Richtwert I) und einen Gefahrenwert (Richtwert II) (s. Abschn. 6). Das Messverfahren sollte den gesamten Bereich der Beurteilungswerte abdecken. Die Anforderungen gemäß DIN EN 482 sind dann entsprechend anzupassen. Die maximal zulässige Messunsicherheit des Messverfahrens von 30\% gilt für den Bereich vom 0,5-Fachen des Richtwertes I bis zum 2-Fachen des Richtwertes II. Für den Konzentrationsbereich vom 0,1 bis zum 0,5 -Fachen des Richtwertes I gilt eine maximal zulässige Messunsicherheit von $50 \%$. Dies gilt für andere Beurteilungsmaßstäbe in analoger Weise.

Bei der Bewertung der Summe flüchtiger organischer Verbindungen (TVOC) sind die oben genannten Anforderungen aufgrund der Vielzahl der zu berücksichtigenden Einzelstoffe in der Regel nicht zu erfüllen, da die Messunsicherheit über die Summe aller Einzelstoffe zu ermitteln ist.

Die Angabe des Messergebnisses sollte der Messunsicherheit der Methode angepasst sein (s. - Tab. 2). Dabei sind die Vorgaben der Ad-hoc-Arbeitsgruppe Innenraumrichtwerte zu berücksichtigen [21].

\subsection{Gase}

\subsubsection{Kohlendioxid}

Die Kohlendioxidkonzentration in der Luft gilt als guter Indikator für die lufthygienischen Bedingungen im Innenraum [22]. Wesentliche Quelle für Kohlendioxid im Innenraum ist der Mensch. Die Überprüfung, ob in einem Raum hinsichtlich der Kohlendioxidkonzentration lufthygienisch unbedenkliche Bedingungen herrschen, hat mit einer kontinuierlichen Messung über den Zeitraum der arbeitstäglichen Nutzung mit einem direkt anzeigenden Messgerät unter normalen Nutzungsbedingungen (vgl. Abschn. 4.3 Messung unter Nutzungsbedingungen) und mit der üblichen Personenbelegung zu erfolgen. Laut ASR A3.6 [4] erfolgt die Messung in einer Höhe von 1,5 $\mathrm{m}$ über dem Fußboden und in einem Abstand von mindestens 1-2 $\mathrm{m}$ von den Wänden entfernt. Für kleinere Räume (bis $50 \mathrm{~m}^{2}$ ) reicht in der Regel eine Probenahmestelle aus. Die Randbedingungen (z. B. Fensteröffnungen, Personenanzahl) sind in der Datenerfassung zu dokumentieren. Während der Messung ist darauf zu achten, dass das Messergebnis nicht 
durch die Atemluft einer in der Nähe befindlichen Person (gilt auch für den Probenehmer) beeinflusst wird. Für die Messungen stehen geeignete infrarotspektrometrische Messgeräte oder elektrochemische Sensoren zur Verfügung. Für orientierende Messungen können auch Prüfröhrchen verwendet werden.

\subsubsection{Stickstoffdioxid}

Stickstoffdioxid $\left(\mathrm{NO}_{2}\right)$ wird an Innenraumarbeitsplätzen in der Regel nicht bestimmt. Falls die Vorermittlung ergeben hat, dass die $\mathrm{NO}_{2}$-Konzentration zu bestimmen ist, so sollte dies nach den Vorgaben der DIN EN ISO 16000-15 erfolgen [23].

\subsubsection{Ozon}

Für die Belastung von Innenräumen mit Ozon ist der Eintrag aus der Außenluft über die Lüftung (z. B. geöffnete Fenster) entscheidend. Die Bildung von Ozon durch den Betrieb von Laserdruckern und Kopierern stellt heute kein Problem mehr dar. Falls die Vorermittlung ergeben hat, dass die Ozonkonzentration zu bestimmen ist, stehen direkt anzeigende Messverfahren zur Verfügung (z. B. chemische Sensoren und UV-Detektoren).

\subsubsection{Radon}

Der größte Teil der Dosis durch natürliche Strahlenquellen wird durch das radioaktive Edelgas Radon und seine Zerfallsprodukte hervorgerufen; auch Baustoffe können zur Belastung beitragen [24]. Eine Übersichtskarte über die Radonkonzentrationen in der Bodenluft ist vom Bundesamt für Strahlenschutz veröffentlicht [25]. Für Arbeitsplätze in Büros und büroähnlichen Räumen besteht entsprechend der EU-Richtlinie 2013/59/EURATOM spätestens ab 2018 eine gesetzliche Verpflichtung zur Ermittlung der Strahlenbelastung durch Radon und zur Einhaltung von Grenzwerten [26]. Die Durchführung von Radonmessungen erfolgt nach der DIN ISO 11665-8 [27].

\subsection{Flüchtige organische Verbindungen}

Zur Bestimmung der VOC gibt es einige gut beschriebene Messverfahren [28, 29, 30, 31, 32], die bei korrekter Anwendung geeignet sind, flüchtige organische Stoffe an Innenraumarbeitsplätzen zu messen. Diese beruhen auf Adsorption der flüchtigen Komponenten an einem Adsorbens. Anschließend werden die VOC thermisch oder mithilfe eines Lösemittels desorbiert. Zur analytischen Bestimmung kommen in der Regel gaschromatographische Verfahren mit massenspektrometrischer oder Flammenionisationsdetektion zum Einsatz.

Es wird empfohlen, zumindest diejenigen VOC als Einzelkomponenten zu kalibrieren, für die ein Innenraumluftrichtwert festgelegt ist. Bei Anwendung der Verfahren erhält man Ergebnisse für diese kalibrierten Einzelkomponenten.

Für Stoffe, bei denen die Konzentration in Bezug auf Toluol berechnet wurde, erhält man das sog. Toluoläquivalent. Aus all diesen Einzelwerten ist die Summe der flüchtigen organischen Verbindungen (total volatile organic compounds TVOC) zu bilden.

\subsubsection{Messung der VOC}

Die Probenahme erfolgt stationär in einer Höhe von 1-1,5 m über dem Fußboden in Atemhöhe der Beschäftigten und in einem Abstand von 1-2 m von den Wänden. Je nach Raumgröße kann es notwendig sein, mehrere Proben an verschiedenen Stellen im Raum zu nehmen. Erfolgt die Innenraummessung aufgrund von Klagen über die Raumluftqualität, ist es sinnvoll, eine Parallelmessung in einem unbelasteten Vergleichsraum (Raum, in dem keine Beschwerden auftreten) durchzuführen, um raumspezifische Unterschiede zu erkennen und mögliche Quellen zu identifizieren. Der Vergleichsraum sollte möglichst in lokaler Nähe liegen und eine vergleichbare Größe und Nutzung zum belasteten Raum aufweisen. In Einzelfällen kann eine Messung der Konzentration an flüchtigen organischen Verbindungen in der Außenluft sinnvoll sein. Sind in mehreren belasteten Räumen Messungen vorgesehen und finden diese Messungen an unterschiedlichen Tagen statt, so wird eine derartige Vergleichsmessung für jeden Messtag empfohlen. Die Außenluftmessung wird in der Nachbarschaft des untersuchten Gebäudes, möglichst in gleicher Höhe, durchgeführt. Ein ausreichender Abstand vom Gebäude ( $>2 \mathrm{~m}$ ) ist anzustreben. Bei
Gebäuden mit RLT-Anlage kann es sinnvoll sein, die Außenluftmessung möglichst in der Nähe der Zuluft durchzuführen.

\subsubsection{Berechnung des TVOC-Wertes}

Bei der Berechnung der TVOC-Konzentration werden nur die Signale berücksichtigt, die oberhalb der Bestimmungsgrenze liegen.

Zunächst sind die Konzentrationen sämtlicher kalibrierter Einzelkomponenten zu berechnen (s. Gl. 1). Für jede kalibrierte Einzelkomponente sollte die Bestimmungsgrenze unter $5 \mu \mathrm{g} / \mathrm{m}^{3}$ liegen.

$$
\rho_{K}=\frac{m_{K}}{V_{\text {Luft }}}
$$

Es bedeuten:

$\rho_{K} \quad$ Massenkonzentration an kalibrierter Einzelkomponente in der Probe in $\mu \mathrm{g} / \mathrm{m}^{3}$

$m_{K} \quad$ Masse an kalibrierter Einzelkomponente in der gesammelten Probe in ng

$V_{\text {Luft }}$ Probeluftvolumen in Liter

Im zweiten Schritt sind alle Konzentrationen der nicht kalibrierten Einzelkomponenten mit Bezug auf Toluol zu berechnen (s. Gl. 2). Diese Stoffe sind ab einem Toluoläquivalent von $1 \mu \mathrm{g} / \mathrm{m}^{3}$ bei der Berechnung zu berücksichtigen.

$$
\rho_{N K}=\frac{m_{N K}}{V_{L u f t}}
$$

Es bedeuten:

$\rho_{N K} \quad$ Massenkonzentration an nicht kalibrierter Einzelkomponente in der Probe in $\mu \mathrm{g} / \mathrm{m}^{3}$

$m_{N K} \quad$ Masse an nicht kalibrierter Einzelkomponente in der gesammelten Probe in ng bezogen auf Toluol

$V_{\text {Luft }}$ Probeluftvolumen in Liter

Der TVOC-Wert setzt sich zusammen aus der Summe der Konzentrationen an kalibrierten und nicht kalibrierten Einzelkomponenten im Retentionsbereich von n-Hexan bis n-Hexadecan (s. Gl. 3).

$$
\begin{aligned}
\rho_{T V O C}= & \sum_{N}^{i}\left(\rho_{\kappa}>B G\right) \\
& +\sum_{N}^{i}\left(\rho_{N K}>1 \mu g / m^{3}\right)
\end{aligned}
$$

Es bedeuten: 
$\rho_{\text {TVOC }}$ Massenkonzentration an TVOC in der Probe in $\mu \mathrm{g} / \mathrm{m}^{3}$

BG Bestimmungsgrenze

Die Zielsetzung von Innenraumuntersuchungen an Arbeitsplätzen ist die Beurteilung von Arbeitsplatzsituationen im Rahmen der Gefährdungsbeurteilung. Für die Bewertung von Innenräumen liegen zahlreiche Richt- bzw. Leitwerte vor, die einzeln zu quantifizieren sind. Dies ist nur mit einer stoffbezogenen Kalibrierung gewährleistet. Hinzu kommt, dass die Erstellung der TVOC-Leitwerte auf der Basis des durch Gl. 3 beschriebenen Verfahrens beruht.

Die hier beschriebene Verfahrensweise zur Berechnung der TVOC-Konzentration weicht von der Vorgehensweise nach DIN ISO 16000-6 ab, weil die vereinfachte TVOC-Berechnung anhand des Toluoläquivalentes für alle VOC gemäß Anmerkung 2 der Norm nur halbquantitativ ist [28].

\subsubsection{Aldehyde}

Aldehyde stellen eine wichtige Stoffgruppe in Innenräumen dar. Auch für Aldehyde gibt es geeignete und gut beschriebene Messverfahren [33, 34, 35]. Die Messverfahren beruhen auf der Sammlung und Derivatisierung von Aldehyden mit anschließender flüssigkeitschromatographischer Bestimmung. Die höheren Aldehyde $\left(>\mathrm{C}_{6}\right)$ werden mit dem oben unter VOC beschriebenen Verfahren bestimmt.

Sollen Aldehyde und VOC am gleichen Arbeitsplatz bestimmt werden, ist zu beachten, dass sich die Messungen unter Umständen beeinflussen können. Bei der Herstellung der Probenträger für Aldehyde wird Acetonitril als Lösemittel verwendet, von dem Reste nach der Herstellung auf den Probenträgern verbleiben können. In ungünstigen Fällen kann das überschüssige Acetonitril während der Sammlung von Aldehyden verdampfen. Es gilt $\mathrm{zu}$ verhindern, dass dieses Acetonitril bei der Probenahme der VOC mit erfasst wird.

\subsection{Schwerflüchtige organische Verbindungen}

Folgende Stoffgruppen sollten nur in begründeten Verdachtsfällen in das Messprogramm mit aufgenommen werden:

\subsubsection{Polycyclische aromatische Kohlenwasserstoffe (PAH)}

Für die Bestimmung von $\mathrm{PAH}$ werden die Messstrategie und das Messverfahren gemäß DIN EN ISO 16000-12 [36] empfohlen.

\subsubsection{Pentachlorphenol (PCP)/Lindan}

Für die Bestimmung von PCP und Lindan werden die Messstrategie und das Messverfahren gemäß VDI 4300-4 [37] und VDI 4301-2 und $3[38,39]$ empfohlen.

\subsubsection{Polychlorierte Biphenyle (PCB)}

Für die Bestimmung von PCB werden die Messstrategie und die Messverfahren gemäß DIN EN ISO 16000-12, -13 und -14 empfohlen [36, 40, 41].

\subsubsection{Weichmacher/ Flammschutzmittel}

Als Weichmacher und Flammschutzmittel wird eine Vielzahl von Stoffen und Stoffgruppen eingesetzt. Für einzelne Stoffe und Stoffgruppen sind Messverfahren verfügbar, wie $z$. B. Flammschutzmittel auf phosphororganischer Basis (VDI 4301-5 [42]) und Phthalate (VDI 4301$6[43])$.

\subsection{Stäube und Fasern}

\subsubsection{Stäube}

Quellen für Stäube in Innenräumen sind die Außenluft, Anhaftungen an Schuhen und Kleidung der Raumnutzer, mechanisches Auf- und Verwirbeln von sedimentierten Partikeln (z. B. durch Staubsaugen, Handhabung von Papier), Verbrennungsvorgänge sowie der Einsatz von Arbeitsmitteln. In Abhängigkeit von der Nutzung variieren die Staubkonzentration und -zusammensetzung in Innenräumen stark [44].

Zur Beurteilung möglicher Gesundheitsgefahren durch Staubbelastungen werden von der Partikelgröße abhängige Staubfraktionen herangezogen. Die im Arbeitsschutz üblicherweise verwendeten Fraktionen „einatembarer Staub“ (EFraktion) und „lungengängiger Staub“ (Alveolarfraktion, A-Fraktion) stimmen nicht exakt überein mit den im Bereich des Umweltschutzes gebräuchlichen
Fraktionen $\mathrm{PM}_{10}$ (thorakaler Feinstaub) und $\mathrm{PM}_{2,5}$ (Feinstaub) [45].

Bei der Messung ist die VDI 4300 Blatt 11[46] zu berücksichtigen.

\subsubsection{Fasern}

Faserförmige Stäube sind gesondert zu betrachten. In Innenräumen sind dies vornehmlich textile Fasern z. B. von Raumtextilien oder Kleidung sowie natürliche oder künstliche Mineralfasern (KMF) z. B. aus thermischen Isolierungen.

Des Weiteren können auch Asbestfasern in Innenräumen auftreten, z. B. in Baumaterialien (Asbestzement), Fußbodenbelägen sowie Schall- und Wärmeisolationen. Eine Freisetzung von Asbestfasern ist insbesondere bei Bau- und Sanierungsmaßnahmen möglich. Dabei sind die Vorgaben zur Bewertung der Dringlichkeit von Sanierungsmaßnahmen entsprechend der Asbestrichtlinie [47] und zum Schutz der Beschäftigten sowie Dritter im Zusammenhang mit Sanierungsmaßnahmen entsprechend TRGS 519 [48] zu beachten. Nach Abschluss entsprechender Maßnahmen ist eine Freimessung für Innenräume erforderlich.

Für die Messplanung und Messung sind die Vorgaben gemäß DIN EN ISO 16000-7 zu beachten [49].

\subsection{Luftwechsel}

Die Luftwechselzahl beschreibt den Luftaustausch im Raum und ergibt sich aus dem Zuluftvolumenstrom bezogen auf das Raumvolumen. Ein ausreichender Luftaustausch sorgt für genügend Zufuhr von Frischluft in die Innenräume, einen Abtransport von Schad- und Geruchstoffen und schützt vor Schäden durch zu hohe oder zu geringe Luftfeuchtigkeit. Der Luftaustausch in einem Raum, der nur über freie Lüftung verfügt, erfolgt über Fenster, Türen und Undichtigkeiten in der Gebäudehülle. Anforderungen zur freien sowie zur maschinellen Lüftung liefert die ASR A3.6 [4]. Bei Einhaltung dieser Vorgaben ist in der Regel die Bestimmung der Luftwechselzahl nicht erforderlich. Bei Verdacht auf eine ineffektive Lüftung z. B. in Großraumbüros kann eine Bestimmung der Luftwechselzahl nach VDI 4300 Blatt 7 [50] und DIN EN ISO 12569 [51] hilfreich sein. 


\section{Beurteilungswerte}

Zur Vorgehensweise bei der Beurteilung von chemischen Verunreinigungen der Innenraumluft liegen Übersichtsarbeiten vor $[21,52]$. Die Beurteilung erfolgt üblicherweise anhand von Grenzwerten, Richtwerten und Leitwerten sowie Referenzwerten. Diese Werte unterscheiden sich hinsichtlich ihrer rechtlichen Verbindlichkeit und ihrer gesundheitlichen Bedeutung. Während Grenzwerte rechtlich verbindlich sind, trifft dies für Richt-, Leit- und Referenzwerte nicht zu.

Grenz-, Richt- und Leitwerte weisen eine toxikologische bzw. gesundheitliche Begründung auf. Hingegen stellen Referenzwerte eine statistische Zustandsbeschreibung dar und sind nicht gesundheitlich begründet. Die einzelnen Beurteilungsmaßstäbe werden im Folgenden beschrieben.

\subsection{Grenzwerte}

Laut VDI 6022 Blatt 3 [53] handelt es sich bei einem Grenzwert um einen ,gesetzlich festgelegten Beurteilungswert, der eingehalten und hinreichend sicher unterschritten werden muss". Grenzwerte lassen sich unterteilen in legislative und administrative Grenzwerte:

Legislative Grenzwerte stellen das Ergebnis eines parlamentarischen Prozesses dar, in dem gesundheitliche und wirtschaftliche Aspekte, Fragen der Machbarkeit und des Aufwandes (z. B. in Abhängigkeit von der Bestimmungsgrenze) gegeneinander abgewogen werden [52]. Bislang gibt es nur den Grenzwert für Tetrachlorethen in der Innenraumluft nach der 2. Bundesimmissionsschutzverordnung (BImSchV) [54].

Administrative Grenzwerte binden einerseits die Verwaltung, bestimmte Vorgehensweisen anzuwenden, beschreiben aber auch Verpflichtungen für sog. betroffene Kreise, wie z. B. Gebäudeeigentümer oder Unternehmen [52]. Hierzu zählen u. a. die Technischen Baubestimmungen für Asbest, $\mathrm{PCP}$ oder PCB und die Technischen Regeln für Gefahrstoffe.

\subsection{Richtwerte}

Laut VDI 6022 Blatt 3 [53] ist ein Richtwert ein „toxikologisch abgeleiteter Wert basierend auf geeigneten Erkenntnissen $\mathrm{zu}$ den toxischen Wirkungen und DosisWirkungs-Beziehungen des jeweiligen Stoffs". In Deutschland werden bundeseinheitliche Richtwerte für die Luft von Innenräumen, in denen keine Tätigkeiten mit Gefahrstoffen erfolgen, im Auftrag der Gesundheitsministerkonferenz von der Ad-hoc-Arbeitsgruppe Innenraumrichtwerte der Kommission Innenraumlufthygiene und der Obersten Landesgesundheitsbehörden erarbeitet [55].

Festgelegt werden jeweils ein Richtwert II (Gefahrenwert), bei dessen „Erreichen bzw. Überschreiten unverzüglich Handlungsbedarf besteht, da diese Konzentration geeignet ist, insbesondere bei Daueraufenthalt in den Räumen die Gesundheit empfindlicher Personen ... zu gefährden ", und ein Richtwert I (Vorsorgewert), bis zu dem ,im Rahmen einer Einzelstoffbetrachtung nach gegenwärtigem Kenntnisstand auch bei lebenslanger Exposition von empfindlichen Personen keine gesundheitlichen Beeinträchtigungen zu erwarten sind" [21].

Die Ad-hoc-Arbeitsgruppe veröffentlicht zu allen festgelegten Richtwerten für die Innenraumluft eine ausführliche Begründung im Bundesgesundheitsblatt. Diese sind auf der Internetseite der Adhoc-Arbeitsgruppe Innenraumrichtwerte beim Umweltbundesamt abrufbar.

\subsection{Leitwerte}

Laut VDI 6022 Blatt 3 [53] ist ein Leitwert ein "gesundheitlich-hygienisch begründeter Beurteilungswert eines Stoffes, für den der Kenntnisstand nicht ausreicht, um einen toxikologisch begründeten Richtwert abzuleiten". Die Ad-hocArbeitsgruppe Innenraumrichtwerte hat bisher Leitwerte für TVOC, Kohlendioxid, Feinstaub $\left(\mathrm{PM}_{2,5}\right)$ und ausgewählte Geruchstoffe in der Innenraumluft abgeleitet $[21,44,56,57]$. Diese sind auf der Internetseite der Ad-hoc-Arbeitsgruppe Innenraumrichtwerte beim Umweltbundesamt abrufbar.
Darüber hinaus werden zu Leitwerten auch Beurteilungswerte von internationalen Gremien gezählt, z. B. die Empfehlungen der Weltgesundheitsorganisation. Die Weltgesundheitsorganisation weist ausdrücklich darauf hin, dass ihre Empfehlungen grundsätzlich unverbindlich sind und von den Staaten ihrem jeweiligen Rechtsrahmen entsprechend angepasst werden müssen [58]. Die Weltgesundheitsorganisation hat erstmals im Jahr 2010 für 6 Stoffe bzw. Stoffgruppen Innenraumluftqualitätsleitwerte und für 3 weitere Stoffe bzw. Stoffgruppen Risikokennzahlen veröffentlicht [58].

\subsection{Referenzwerte}

Ein Referenzwert ist „ein Wert, der aus einer Reihe von Messwerten einer Stichprobe aus einer Grundgesamtheit nach einem vorgegebenen Verfahren abgeleitet worden ist. Es handelt sich um einen rein statistisch definierten Wert, der die Verteilung dieses Stoffes im betreffenden Umweltmedium für eine definierte Grundgesamtheit zum Zeitpunkt der Durchführung der Untersuchung beschreibt. Referenzwerte bilden die derzeit allgemein vorhandene Exposition gegenüber einem Stoff („Hintergrundbelastung") ab und geben keinen Aufschluss über eine Gesundheitsgefährdung " [21].

Ein Referenzwert soll ,als ein Zahlenwert angegeben werden, der eindeutig durch das angewandte statistische Verfahren und die zu einem bestimmten Zeitpunkt untersuchte Grundgesamtheit definiert ist. Als (oberer) Referenzwert gilt das 95. Perzentil der Stoffkonzentration in dem für die Referenzpopulation untersuchten Umweltmedium. Die Festlegung auf das 95. Perzentil stellt eine international akzeptierte Konvention dar. Als zusätzliche wichtige Information sollte unbedingt auch die Größe der Stichprobe, aus der der Referenzwert abgeleitet wurde, genannt werden. Die Vertrauenswürdigkeit des statistisch ermittelten 95. Perzentils lässt sich beispielsweise anhand der Angabe des 0,95-Konfidenzintervalls des 95. Perzentils beschreiben" [21].

Referenzwerte liegen u. a. für Büroräume [15] und für Klassenräume [59, 60] vor. Weitere Informationen zu privat, öf- 
fentlich und gewerblich genutzten Innenräumen finden sich in [61].

\subsection{Beurteilung von Messergebnissen}

Für die Beurteilung der Innenraumluft gilt: Grenz-, Richt- und Leitwerte sind grundsätzlich einzuhalten. Bei Richtwerten ist der Vorsorgewert (Richtwert I) heranzuziehen. Werden diese Werte überschritten, sind Maßnahmen entsprechend der Handreichung [21] der Ad-hoc-Ar- beitsgruppe Innenraumrichtwerte zu ergreifen. Die Wirksamkeit dieser Maßnahmen ist zu überprüfen.

Liegen für Stoffe oder Stoffgruppen keine Grenz-, Richt- oder Leitwerte vor, können ggf. vorhandene Referenzwerte herangezogen werden. Bei Überschreitung derartiger Referenzwerte ist zu prüfen, welche Ursache dafür verantwortlich sein kann. Dies kann z. B. durch Vergleich mit der Konzentration in der Außenluft erfolgen (Straßenverkehr, benachbarte Industrie, Wetterlagen). Ebenso sind kürzlich durchgeführte bauliche Maßnahmen oder Instandhaltungsarbeiten $\mathrm{zu}$ berücksichtigen. Auf der Grundlage dieser Informationen ist zu prüfen, ob eine dauerhafte Überschreitung der Referenzwerte oder eine Verringerung der Konzentration zu erwarten ist. Ist eine länger anhaltende Überschreitung eines Referenzwertes zu erwarten, sollte eine weitergehende fachliche Beratung z. B. durch die zuständige Behörde oder den Unfallversicherungsträger eingeholt werden.

\section{Anhang}

Beispiel für eine Checkliste zur Vorermittlung einer Messung an Innenraumarbeitsplätzen

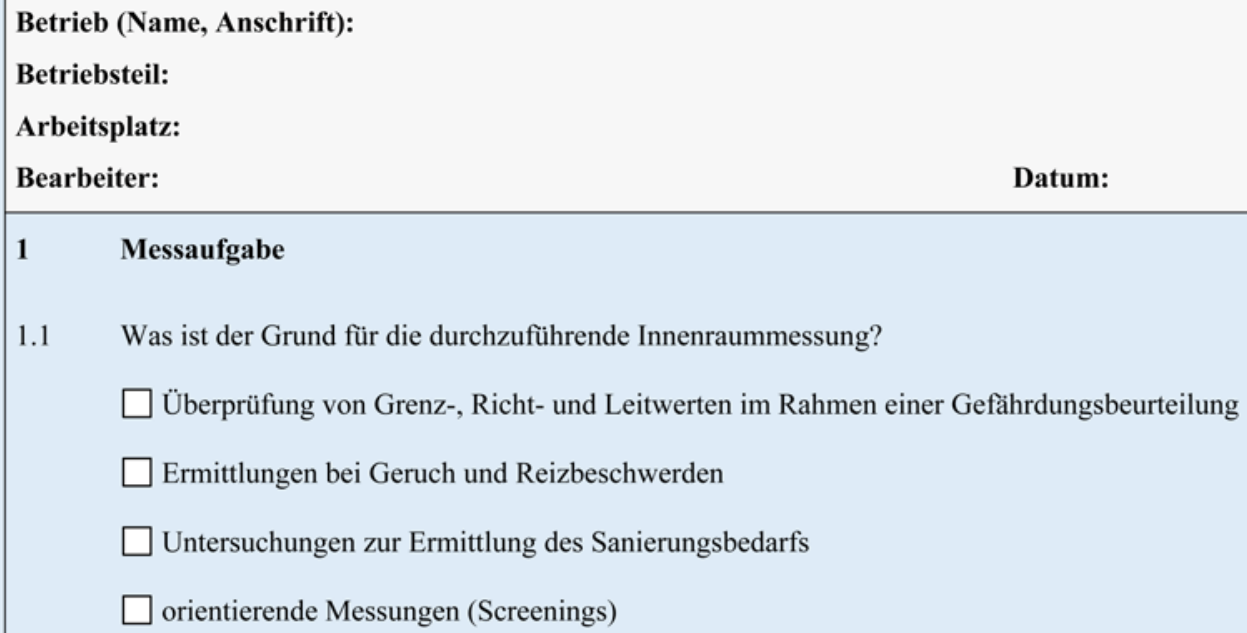

1.1 Was ist der Grund für die durchzuführende Innenraummessung?

Überprüfung von Grenz-, Richt- und Leitwerten im Rahmen einer Gefährdungsbeurteilung

Ermittlungen bei Geruch und Reizbeschwerden

Untersuchungen zur Ermittlung des Sanierungsbedarfs

orientierende Messungen (Screenings)

\section{Angaben zu möglichen Beschwerden der Beschäftigten}

2.1 Wie viele Beschäftigte äußern Beschwerden? Anteil $\%$

2.2 Welche Art von Beschwerden treten auf?

2.3 Seit wann treten die Beschwerden auf?

2.4 Wann treten die Beschwerden auf?

2.5 Können die Beschwerden auf eine der folgenden Ursachen zurückgeführt werden?

hohe Beanspruchung der Beschäftigten

unzureichendes Betriebsklima

Änderungen in der Organisationsstruktur

unzureichende Beleuchtung

störende Geräusche

unzureichendes Raumklima 


\section{Bekanntmachungen - Amtliche Mitteilungen}

störende Gerüche von außen

störende Gerüche im Inneren

$\square$ Renovierungsarbeiten

Sonstiges, und zwar

\section{Allgemeine Gebäudedaten}

3.1 Wann wurde das Gebäude errichtet?

\section{Angaben zur Gebäudelage}

4.1 Wo befindet sich das Gebäude?

$\square$ in der Innenstadt

$\square$ in einem Industrie- oder Gewerbegebiet

$\square$ in einem Mischgebiet

$\square$ in einem Wohngebiet

$\square$ an einer viel befahrenen Straße/Autobahn/Bahntrasse

$\square$ sonstige Lage, und zwar

Fügen Sie, wenn möglich, einen Umgebungsplan oder eine Skizze bei!

4.2 Gibt es Industrieanlagen in unmittelbarer Nähe des Gebäudes?
$\square$ Nein
$\square \mathrm{Ja}$, und zwar

4.3 Sind Abluftkamine/äußere Schadstoffquellen in der Umgebung des Gebäudes bekannt?
Nein
Ja, und zwar

5 Angaben zu den Arbeitsbereichen oder Gebäudeteilen, in denen Beschwerden von Mitarbeitern vorliegen

5.1 Wie werden die Arbeitsbereiche/Gebäudeteile genutzt?

\begin{tabular}{|l|l|l|l|}
\hline $\begin{array}{l}\text { Arbeitsbereich/ } \\
\text { Gebäudeteil }\end{array}$ & $\begin{array}{l}\text { Größe der } \\
\text { Räume }\end{array}$ & $\begin{array}{l}\text { Art der Nutzung } \\
\text { (z. B. Bildschirmarbeitsplatz) }\end{array}$ & Bemerkungen \\
\hline & & & \\
\hline & & & \\
\hline
\end{tabular}

5.2 Wurden Arbeitsbereiche/umliegende Gebäudeteile in der Vergangenheit anders genutzt?

Nein

$\mathrm{Ja}$

Bitte tragen Sie Art und Zeitraum der Nutzung in die folgende Tabelle ein.

\begin{tabular}{|l|l|l|l|}
\hline $\begin{array}{l}\text { Arbeitsbereich/ } \\
\text { Gebäudeteil }\end{array}$ & Art der Nutzung & Zeitraum & Bemerkungen \\
\hline & & & \\
\hline & & & \\
\hline
\end{tabular}


5.3 Wurden Gebäuderenovierungen, An- oder Umbauten vorgenommen?

$\square$ Nein

$\square \mathrm{Ja}$

Bitte tragen Sie Art und Umfang der Änderungen in die folgende Tabelle ein.

\begin{tabular}{|l|l|}
\hline Zeitraum & $\begin{array}{l}\text { Art und Umfang der Gebäudeänderungen } \\
\text { (z. B. Anstriche, Bodenverlegungen, zusätzliche Fenster, } \\
\text { Wärmedämmung, Abdichtungen, Asbestsanierung) }\end{array}$ \\
\hline & \\
\hline & \\
\hline
\end{tabular}

6 Angaben zur Gebäudelüftung

6.1 Werden die Räume natürlich (über Fenster) belüftet?

$\square$ Nein $\square$ Ja

Bemerkungen:

6.2 Geben Sie die Art der Fenster an.

$\square$ Holz

$\square$ Metall

$\square$ Kunststoff

$\square$ Sonstige, und zwar

6.3 Werden die Räume technisch belüftet?

$\square$ Nein

$\square$ Nur technische Zu- und Abluft

$\square$ Klimaanlage

Mit Luftbefeuchtung

Bemerkungen:

6.4 Falls vorhanden: Wird die Lüftungs-/Klimaanlage regelmäßig überprüft?
$\square$ Nein
$\mathrm{Ja}$, durch

$\square$ Dokumentation liegt vor

7 Welcher Art ist die Heizungsanlage?

$\square$ Gas

$\square$ Öl

$\square$ Sonstige, und zwar

8 Bauart und Baustoffe der Decken in den betroffenen Arbeitsräumen

8.1 Bauweise

$\square$ Beton

$\square$ Estrich

$\square$ Holz

$\square$ Faserplatte

$\square$ Sonstiges, und zwar

8.2 Geben Sie die Art der unteren Deckenkonstruktion an.

Abgehängte Decke

Akustikdecke

$\square$ Lüftungsdecke 
$\square$ Verputzte Decke

$\square$ Sonstige Konstruktion, und zwar

8.3 Beschreiben Sie die Verkleidung der Decken in den betroffenen Arbeitsräumen.

$\square$ Papiertapete

$\square$ Kunststofftapete

Putz mit Anstrich

Beschichtete Platten

Holzverkleidung

Sonstige Verkleidung, und zwar

8.4 Geben Sie die Art der Oberflächenbehandlung der Decke an.

Keine Oberflächenbehandlung

Gestrichen

Sonstige Behandlung, und zwar

9 Bauart und Baustoffe der Wände in den betroffenen Arbeitsräumen

Bei unterschiedlichen Bauarten und Baustoffen ist der folgende Teil für jeden Arbeitsraum getrennt aufzuführen.

$9.1 \quad$ Bauweise

Fertigbauweise (z. B. Holzständerbauweise)

$\square$ Betonfertigplatten mit dauerelastischem Fugenmaterial

$\square$ Mauerwerksbau unter Verwendung von

$\square$ Kalksandsteinen

$\square$ Ziegeln

Bims

$\square$ Gasbeton

$\square$ Sonstigem, und zwar

$\square$ Holzbau

Containerbauweise

Sonstige Bauweise, und zwar

9.2 Sind die Außenwände wärmegedämmt?

$\square$ Nein $\square$ Ja, und zwar z. B. Innen- oder Kerndämmung; Dämmstoff

9.3 Geben Sie die Art der Zwischen- und Trennwände an.

$\square$ Gemauert

$\square$ Trockenwand

Sonstige, und zwar

9.4 Beschreiben Sie die Verkleidung der Wände in den betroffenen Arbeitsräumen.

$\square$ Papiertapete

Kunststofftapete

Putz mit Anstrich

Beschichtete Platten

Holzverkleidung

Sonstige Verkleidung, und zwar 
9.5 Geben Sie die Art der Oberflächenbehandlung der Wände an.

$\square$ Keine Oberflächenbehandlung

$\square$ Gestrichen

$\square$ Sonstige Behandlung, und zwar

\section{Fußboden}

10.1 Beschreiben Sie den Fußboden in den betroffenen Arbeitsräumen.

$\square$ Parkett, und zwar Parkettart

Vorstrich: Handelsname

Kleber: Handelsname

Versiegelung: Handelsname

Holzdielen, und zwar

Vorstrich: Handelsname

Kleber: Handelsname

Versiegelung: Handelsname

Teppichboden, und zwar Material

verklebt: $\square$ Nein $\square$ Ja, und zwar mit Kleber

Alter: Jahre

Kunststoffbelag, und zwar Material

verklebt: $\square$ Nein $\square$ Ja, und zwar mit Kleber

Alter: Jahre

Fliesen, und zwar Material

verklebt: $\square$ Nein $\square$ Ja, und zwar mit Kleber

Alter: Jahre

Sonstiger Belag, und zwar

10.2 Trägt der Fußbodenbelag ein Gütesiegel?
$\square$ Nein
$\mathrm{Ja}$, und zwar

10.3 Geben Sie den GISCODE des Verlegewerkstoffes an.

10.4 Sicherheitsdatenblätter des Verlegewerkstoffes

$\square$ Liegen bei

$\square$ Werden beschafft

Sind nicht beschaffbar

11 Sind in den betroffenen Arbeitsräumen Dichtungsfugen sichtbar?
$\square$ Nein
$\mathrm{Ja}$

12 Möbel

12.1 Wie alt sind die Möbel in den betroffenen Arbeitsräumen durchschnittlich?

Jahre

12.2 Sind innerhalb des letzten Jahres neue Möbel in dem betroffenen Arbeitsraum aufgestellt worden?
$\square$ Nein
Ja, und zwar
vor
Monaten 
12.3 Aus welchem Material bestehen die Möbel?

$\square$ Massivholz

Holzwerkstoffe

$\square$ Spanplatten

$\square$ Tischlerplatten

$\square$ Furnierplatten

$\square$ Faserplatten

Metall

Kunststoff

Materialkombinationen, und zwar

Sonstiges, und zwar

12.4 Besitzen die Möbelstücke eine Kennzeichnung?

\section{$\square$ Möbelpass}

$\square$ Umweltzeichen „Blauer Engel“

$\square$ RAL-Gütezeichen

$\square$ Sonstige Kennzeichnung, und zwar

Keine Kennzeichnung

\section{Raumtextilien}

13.1 Sind in den betroffenen Arbeitsräumen Polstermöbel vorhanden?
$\square$ Nein
Ja, und zwar Anzahl und Art

13.2 Sind in den betroffenen Arbeitsräumen Gardinen bzw. Stores vorhanden?
$\square$ Nein
Ja, und zwar Anzahl und Art

13.3 Sind in den betroffenen Arbeitsräumen großflächige textile Objekte vorhanden?

$\square$ Nein

Ja, und zwar Anzahl und Art

\section{Angaben zur technischen Ausstattung der Arbeitsbereiche}

14.2 Befinden sich Geräte, Maschinen oder andere Einrichtungen (z. B. Drucker, Kopierer) im Arbeitsbereich, von denen störende Emissionen (z. B. Lärm, Gerüche) ausgehen?
Nein
Ja, und zwar Anzahl und Art

14.3 Werden diese Betriebseinrichtungen regelmäßig gewartet und geprüft?
Nein
Ja, durch

$\square$ Dokumentation liegt vor

15 Befinden sich Pflanzen im Arbeitsraum?
Nein
Ja, und zwar Art

Anzahl:

Kultivierung: z. B. Erde, Hydrokultur

16 Reinigung und Pflege der betroffenen Räume

16.1 Wann werden die Räume gereinigt/gepflegt?

\section{Täglich}

Wöchentlich

Monatlich

Jährlich

Anderer Zeitraum, und zwar 
16.2 Womit werden die Räume gereinigt/gepflegt?

16.3 Werden oder wurden im Raum weitere Produkte verwendet?

$\square$ Nein $\square$ Ja

$\square$ Desinfektionsmittel

$\square$ Raumluftverbesserer

Insektizide, Fungizide, Schädlingsbekämpfungsmittel

Sonstige, und zwar

\section{Liegen bereits ältere Raumluftmessergebnisse vor?}

Nein

$\mathrm{Ja}$, und zwar

Formaldehyd:

$\mu \mathrm{g} / \mathrm{m}^{3}$

VOC: $\quad \mu \mathrm{g} / \mathrm{m}^{3}$

Sonstige Gefahrstoffe,

und zwar Gefahrstoff und Messergebnis

\section{Anmerkungen}

Diese Mitteilung wurde von Dr. Dietmar Breuer, Dr. Helmut Sagunski, Dr. Michael Ball, Dr. Ralf Hebisch, Dr. Nadja von Hahn, Herrn Thomas Lahrz, Dr. Gerda Nitz, Herrn Karl-Heinz Pannwitz und Herrn Wolfgang Rosenberger erstellt und von der Ad-hoc-Arbeitsgruppe Innenraumrichtwerte im Dezember 2013 und von der Arbeitsgruppe Luftanalysen im April 2014 verabschiedet.

\section{Literatur}

1. Verordnung zum Schutz vor Gefahrstoffen (Gefahrstoffverordnung - GefStoffV) vom 26. November 2010. BGBI. (2010) Nr. 59, S. 1643, zul. geänd. BGBI. (2013), S 944

2. Technische Regeln für Gefahrstoffe: TRGS 900 Arbeitsplatzgrenzwerte (2006); zuletzt geändert und ergänzt: GMBI 2013 S. 943-947 vom 19.09.2013 [Nr. 47]. http://www.baua.de/de/Themen-von-A-Z/Gefahrstoffe/TRGS/TRGS-900.htm

3. Deutsche Forschungsgemeinschaft (2013) MAKund BAT-Werte-Liste 2013. Senatskommission zur Prüfung gesundheitsschädlicher Arbeitsstoffe, Mitteilung 49, Wiley-VCH, Weinheim. http://onlinelibrary.wiley.com/book/10.1002/9783527675135

4. Technische Regeln für Arbeitsstätten: Lüftung (ASR A3.6). GMBI. (2012) Nr. 6, S. 92-97, geänd. GMBI. (2013), S. 359. http://www.baua.de/de/Themenvon-A-Z/Arbeitsstaetten/ASR/ASR-A3-6.html

5. DIN EN ISO 16000-1 (2006) Innenraumluftverunreinigungen - Teil 1: Allgemeine Aspekte der Probenahmestrategie. Beuth, Berlin

6. Verordnung über Arbeitsstätten (Arbeitsstättenverordnung - ArbStättV) vom 12. August 2004. BGBI. (2004), S 2179, zul. geänd. BGBI. (2010), S 960

7. Innenraumarbeitsplätze - Vorgehensempfehlung für die Ermittlungen zum Arbeitsumfeld, Report der gewerblichen Berufsgenossenschaften, der Unfallversicherungsträger der öffentlichen Hand und des Instituts für Arbeitsschutz der Deutschen Gesetzlichen Unfallversicherung (IFA), Hrsg.: Deutsche Gesetzliche Unfallversicherung (DGUV), Berlin (2013), http://publikationen.dguv.de/dguv/ pdf/10002/rep_ira.pdf
8. Analytische Methoden zur Prüfung gesundheitsschädlicher Arbeitsstoffe: Luftanalysen. Arbeitsgruppe "Analytische Chemie" der Kommission zur Prüfung gesundheitsschädlicher Arbeitsstoffe der DFG, Wiley-VCH. http://onlinelibrary.wiley.com/ book/10.1002/3527600418/topics

9. Technische Regeln für Gefahrstoffe: Ermitteln und Beurteilen der Gefährdungen bei Tätigkeiten mit Gefahrstoffen: Inhalative Exposition (TRGS 402). GMBI. (2010), Nr. 12, S 231, geänd. GMBI. (2011), $\mathrm{S} 172$

10. DIN EN ISO 16000 Reihe: Innenraumluftverunreinigungen. Beuth, Berlin

11. VDI 4300 Reihe, Messen von Innenraumluftverunreinigungen. Beuth, Berlin

12. Vorgehensweise bei der gesundheitlichen Bewertung der Emissionen von flüchtigen organischen Verbindungen (VOC und SVOC) aus Bauprodukten, Ausschuss zur gesundheitlichen Bewertung von Bauprodukten (AgBB), Juni 2012. http://www. umweltbundesamt.de/sites/default/files/medien/ pdfs/agbb_bewertungsschema_2012.pdf

13. Seifert B, Ullrich D (1987) Methodologies for evaluating sources of VOC in homes. Atmosph Environm 21:395-404

14. Ad-hoc-Arbeitsgruppe Innenraumrichtwerte der IRK/AOLG: Richtwerte für die Innenraumluft. http://www.umweltbundesamt.de/themen/gesundheit/kommissionen-arbeitsgruppen/ad-hocarbeitsgruppe-innenraumrichtwerte

15. von Hahn N et al (2011) Ableitung von Innenraumarbeitsplatz-Referenzwerten. Gefahrstoffe - Reinhaltung der Luft 71(7/8):314-322

16. DIN EN 482 (2012) Exposition am Arbeitsplatz Allgemeine Anforderungen an die Leistungsfähigkeit von Verfahren zur Messung chemischer Arbeitsstoffe (06.12). Beuth, Berlin

17. DIN EN 838 (2010) Exposition am Arbeitsplatz Messung von Gasen und Dämpfen mit Diffusionssammlern - Anforderungen und Prüfverfahren. Beuth, Berlin

18. DIN EN 1076 (2010) Exposition am Arbeitsplatz Messung von Gasen und Dämpfen mit pumpenbetriebenen Probenahmeeinrichtungen - Anforderungen und Prüfverfahren (04.10). Beuth, Berlin
19. DIN EN 13890 (2010) Exposition am Arbeitsplatz Messung von Metallen und Metalloiden in luftgetragenen Partikeln - Anforderungen und Prüfverfahren (01.10). Beuth, Berlin

20. prEN 13936 (2012) Exposition am Arbeitsplatz Messung eines als Mischung aus luftgetragenen Partikeln und Dampf vorliegenden chemischen Arbeitsstoffes - Anforderungen und Prüfverfahren. Beuth, Berlin

21. Ad-hoc-Arbeitsgruppe Innenraumrichtwerte der IRK/AOLG (2007) Beurteilung von Innenraumluftkontaminationen mittels Referenz- und Richtwerten. Bundesgesundheitsbl Gesundheitsforsch Gesundheitsschutz 50(7):990-1005. http://www.umweltbundesamt.de/sites/default/files/medien/ pdfs/Handreichung.pdf

22. DIN EN ISO 16000-26 (2012) Innenraumluftverunreinigungen - Teil 26: Probenahmestrategie für Kohlendioxid $\left(\mathrm{CO}_{2}\right)$. Beuth, Berlin

23. DIN EN ISO 16000-15 (2009) Innenraumluftverunreinigungen - Teil 15: Probenahmestrategie für Stickstoffdioxid $\left(\mathrm{NO}_{2}\right)$. Beuth, Berlin

24. Natürliche Radionuklide in Baumaterialien. http:// www.bfs.de/de/ion/anthropg/baustoffe.html

25. http://www.bfs.de/de/ion/anthropg/radon/radon_boden/radonkarte.html

26. RICHTLINIE 2013/59/EURATOM DES RATES vom 5. Dezember 2013 zur Festlegung grundlegender Sicherheitsnormen für den Schutz vor den Gefahren einer Exposition gegenüber ionisierender Strahlung und zur Aufhebung der Richtlinien 89/618/ Euratom, 90/641/Euratom, 96/29/Euratom, 97/43/ Euratom und 2003/122/Euratom. Amtsbl. Europ. Union vom 17.01.2014, L13:1-73

27. DIN ISO 11665-8 (2013) Ermittlung der Radioaktivität in der Umwelt-Luft: Radon-222 - Teil 8: Methodik zur Erstbewertung sowie für zusätzliche Untersuchungen in Gebäuden (08.13). Beuth, Berlin

28. DIN ISO 16000-6 (2012) Innenraumluftverunreinigungen - Teil 6: Bestimmung von VOC in der Innenraumluft und in Prüfkammern, Probenahme auf Tenax TA, thermische Desorption und Gaschromatographie mit MS/FID (11.12). Beuth, Berlin 
29. DIN EN ISO 16017-1 (2001) Innenraumluft, Außenluft und Luft am Arbeitsplatz - Probenahme und Analyse flüchtiger organischer Verbindungen durch Sorptionsröhrchen/thermische Desorption/ Kapillar-Gaschromatographie - Teil 1: Probenahme mit einer Pumpe. Beuth, Berlin

30. DIN EN ISO 16017-2 (2003) Innenraumluft, Außenluft und Luft am Arbeitsplatz - Probenahme und Analyse flüchtiger organischer Verbindungen durch Sorptionsröhrchen/thermische Desorption/ Kapillar-Gaschromatographie - Teil 2: Probenahme mit Passivsammlern. Beuth, Berlin

31. VDI 2100-2 (2011) Messen gasförmiger Verbindungen in der Außenluft - Messen von Innenraumluftverunreinigungen - Gaschromatografische Bestimmung organischer Verbindungen - Aktive Probenahme durch Anreicherung auf Aktivkohle Lösemittelextraktion. Beuth, Berlin

32. Breuer D, Friedrich C, Moritz A. (1989) VOC (Volatile Organic Compounds, flüchtige organische Verbindungen) (Kennzahl 8936). In: Institut für Arbeitsschutz der Deutschen Gesetzlichen Unfallversicherung (IFA), Sankt Augustin (Hrsg) IFA-Arbeitsmappe Messung von Gefahrstoffen. 45. Lfg. X/10. Erich Schmidt - Losebl.-Ausg, Berlin. www.ifa-arbeitsmappedigital.de/8936

33. DIN EN ISO 16000-2 (2006) Innenraumluftverunreinigungen - Teil 2: Probenahmestrategie für Formaldehyd. Beuth, Berlin

34. DIN ISO 16000-3 (2013) Innenraumluftverunreinigungen - Teil 3: Messen von Formaldehyd und anderen Carbonylverbindungen in der Innenraumluft und in Prüfkammern - Probenahme mit einer Pumpe. Beuth, Berlin

35. DIN ISO 16000-4 (2012) Innenraumluftverunreinigungen - Teil 4: Bestimmung von Formaldehyd Probenahme mit Passivsammlern. Beuth, Berlin

36. DIN EN ISO 16000-12 (2008) Innenraumluftverunreinigungen - Teil 12: Probenahmestrategie für polychlorierte Biphenyle (PCB), polychlorierte Dibenzo-p-dioxine (PCDD), polychlorierte Dibenzofurane (PCDF) und polycyclische aromatische Kohlenwasserstoffe (PAH). Beuth, Berlin

37. VDI 4300-4 (1997) Messen von Innenraumluftverunreinigungen - Meßstrategie für Pentachlorphenol (PCP) und $\gamma$-Hexachlorcyclohexan (Lindan) in der Innenraumluft. Beuth, Berlin

38. VDI 4301-2 (2000) Messen von Innenraumluftverunreinigungen - Messen von Pentachlorphenol (PCP) und $\gamma$-Hexachlorcyclohexan (Lindan) - GC/ MS-Verfahren. Beuth, Berlin

39. VDI 4301-3 (2003) Messen von Innenraumluftverunreinigungen - Messen von Pentachlorphenol (PCP) und $\gamma$-Hexachlorcyclohexan (Lindan) - GC) ECD-Verfahren (06.03). Beuth, Berlin

40. DIN ISO 16000-13 (2010) Innenraumluftverunreinigungen - Teil 13: Bestimmung der Summe gasförmiger und partikelgebundener dioxin-ähnlicher Biphenyle (PCB) und polychlorierter Dibenzo-p-dioxine/Dibenzofurane (PCDD/PCDF) - Probenahme auf Filtern mit nachgeschalteten Sorbenzien. Beuth, Berlin

41. DIN ISO 16000-14 (2012) Innenraumluftverunreinigungen - Teil 14: Bestimmung der Summe gasförmiger und partikelgebundener polychlorierter dioxin-ähnlicher Biphenyle (PCB) und polychlorierter Dibenzo-p-dioxine/Dibenzofurane (PCDD/PCDF) Extraktion, Reinigung und Analyse mit hochauflösender Gaschromatographie/Massenspektrometrie. Beuth, Berlin
42. VDI 4301-5 (2009) Messen von Innenraumluftverunreinigungen - Messen von Flammschutzmitteln und Weichmachern auf Basis phosphororganischer Verbindungen - Phosphorsäureester. Beuth, Berlin

43. VDI 4301-6 (2012) Messen von Innenraumluftverunreinigungen - Messen von Phthalaten mit GC/ MS. Beuth, Berlin

44. Ad-hoc-Arbeitsgruppe Innenraumrichtwerte der IRK/AOLG (2008) Gesundheitliche Bedeutung von Feinstaub in der Innenraumluft. Bundesgesundheitsbl Gesundheitsforsch Gesundheitsschutz 51(7):1370-1378. http://www.umweltbundesamt. de/themen/gesundheit/kommissionen-arbeitsgruppen/ad-hoc-arbeitsgruppe-innenraumrichtwerte

45. Mattenklott M, Höfert N (2009) Stäube an Arbeitsplätzen und in der Umwelt - Vergleich der Begriffsbestimmungen. Gefahrstoffe - Reinhaltung der Luft 69(4):127-129

46. VDI 4300-11 (2011) Messen von Innenraumluftverunreinigungen - Messstrategie für die Erfassung von luftgetragenen Partikeln im Innenraum - Partikel mit Äquivalentdurchmesser $<2,5 \mu \mathrm{m}$. Beuth, Berlin

47. Richtlinie für die Bewertung und Sanierung schwach gebundener Asbestprodukte in Gebäuden (Asbest-Richtlinie) (01.96). Deutsches Institut für Bautechnik. DIBt-Mitteilungen Heft 3/1996, S 88

48. Technische Regeln für Gefahrstoffe: Asbest Abbruch-, Sanierungs- oder Instandhaltungsarbeiten (TRGS 519). GMBI. (2007) Nr. 6/7, S 122, ber. Seite 398

49. DIN EN ISO 16000-7 (2007) Innenraumluftverunreinigungen - Teil 7: Probenahmestrategie zur Bestimmung luftgetragener Asbestfaserkonzentrationen. Beuth, Berlin

50. VDI 4300 Blatt 7 (2001) Bestimmung der Luftwechselzahl in Innenräumen. Beuth, Berlin

51. DIN EN ISO 12569 (2010) Wärmetechnisches Verhalten von Gebäuden und Werkstoffen - Bestimmung des spezifischen Luftvolumenstroms in $\mathrm{Ge}$ bäuden - Indikatorgasverfahren. Beuth, Berlin

52. Sagunski H, Heinzow B, Müller K (2013) Referenzwerte, Leitwerte, Richtwerte und Grenzwerte für die Innenraumluft - eine Kurzanleitung. Gefahrstoffe - Reinhaltung der Luft 73(4):169-175

53. VDI 6022 Blatt 3 (2011) Raumlufttechnik - Raumluftqualität - Beurteilung der Raumluftqualität. Beuth, Berlin

54. Zweite Verordnung zur Durchführung des BundesImmissionsschutzgesetzes (2. BImSchV) - Verordnung zur Emissionsbegrenzung von leichtflüchtigen halogenierten organischen Verbindungen vom 10. Dezember 1990. BGBI. I, S. 2694; zul. geänd. BGBI. I (2013), S. 1021. http://www.gesetzeim-internet.de/bundesrecht/bimschv_2_1990/gesamt.pdf

55. Ad-hoc-Arbeitsgruppe Innenraumrichtwerte der IRK/AOLG (2012) Richtwerte für die Innenraumluft: erste Fortschreibung des Basisschemas. Bundesgesundheitsbl Gesundheitsforsch Gesundheitsschutz 55(2):279-290. http://www.umweltbundesamt.de/themen/gesundheit/kommissionen-arbeitsgruppen/ad-hoc-arbeitsgruppe-innenraumrichtwerte
56. Ad-hoc-Arbeitsgruppe Innenraumrichtwerte der IRK/AOLG (2008) Gesundheitliche Bewertung von Kohlendioxid in der Innenraumluft. Bundesgesundheitsbl Gesundheitsforsch Gesundheitsschutz 51(11):1358-1369. http://www.umweltbundesamt.de/themen/gesundheit/kommissionen-arbeitsgruppen/ad-hoc-arbeitsgruppe-innenraumrichtwerte

57. Ad-hoc-Arbeitsgruppe Innenraumrichtwerte der IRK/AOLG (2014) Gesundheitlich-hygienische Bewertung von Geruchsstoffen in der Innenraumluft mit Hilfe von Geruchsleitwerten. Bundesgesundheitsbl Gesundheitsforsch Gesundheitsschutz 57(1):148-153. http://www.umweltbundesamt. de/themen/gesundheit/kommissionen-arbeitsgruppen/ad-hoc-arbeitsgruppe-innenraumrichtwerte

58. WHO guidelines for indoor air quality: selected pollutants. Hrsg.: Weltgesundheitsorganisation, Genf 2010

59. Ostendorp G, Riemer D, Harmel K, Heinzow B (2009) Aktuelle Hintergrundwerte zur VOC-Belastung in Schulen und Kindergärten in Schleswig-Holstein. Umweltmedizin Forschung Praxis 14(3):135-152

60. Neumann HD, Buxtrup M, von Hahn N, Koppisch D, Breuer D, Hahn J-U (2012) Vorschlag zur Ableitung von Innenraumarbeitsplatz-Referenzwerten in Schulen. Gefahrstoffe - Reinhaltung der Luft 72(7/8):291

61. Hofmann $\mathrm{H}$, Plieninger $\mathrm{P}$ (2008) Bereitstellung einer Datenbank zum Vorkommen von flüchtigen organischen Verbindungen in der Raumluft. Umweltbundesamt. Forschungsbericht 20561 234. UBA-FB 001131. http://www.umweltbundesamt.de/sites/default/files/medien/publikation/ long/3637.pdf 